\title{
Enhanced Magnetic Reconnection in the Presence of Pressure Gradients
}

\author{
M.J. Pueschel ${ }^{1}$, P.W. Terry ${ }^{1}$, D. Told ${ }^{2,3}$, and F. Jenko ${ }^{2,3}$ \\ 1 Department of Physics, University of \\ Wisconsin-Madison, Madison, Wisconsin 53706, USA \\ 2 Max-Planck-Institut für Plasmaphysik, D-85748 Garching, Germany \\ 3 Department of Physics and Astronomy, \\ University of California, Los Angeles, California 90095, USA
}

\begin{abstract}
Magnetic reconnection in the presence of background pressure gradients is studied, with special attention to parallel (compressional) magnetic fluctuations. A process is reported that reconnects fields through coupling of drift-wave-type instabilities with current sheets. Its time scale is set not by the reconnecting field but by inhomogeneities of the background density or temperature. The observed features can be attributed to a pressure-gradient-driven linear instability which interacts with the reconnecting system but is fundamentally different from microtearing. In particular, this mode relies on parallel magnetic fluctuations and the associated drift. For turbulent reconnection, similar or even stronger enhancements are reported. In the solar corona, this yields a critical pressure gradient scale length of about $200 \mathrm{~km}$ below which this new process becomes dominant over the tearing instability.
\end{abstract}




\section{INTRODUCTION}

In the space plasma community, magnetic reconnection driven by current gradients - i.e., tearing modes - is an area receiving significant attention [1-6]. Meanwhile fusion researchers have long investigated tearing mode physics (see, e.g., Ref. [7]), and recently important strides have been made in advancing our knowledge of microtearing (MT) modes [8-10], which drive reconnection by background electron temperature gradients.

Some studies have focused on the impact of density and temperature gradients on collisionless tearing (CT) modes [11-14], where in the standard picture, such gradients create stabilizing diamagnetic flows which reduce the growth rate $\gamma$ relative to its gradient-free value $\gamma_{0}$ (note that the electron temperature gradient may also have an impact, see Ref. [15]). For strong guide fields and neglecting parallel magnetic fluctuations $B_{\|}$, this approach (see, e.g., Refs. $[12,16])$ predicts

$$
\gamma^{2} \approx \gamma_{0}^{2}-\frac{k_{y}^{2} \omega_{n}^{2}}{4}\left(1+\frac{T_{\mathrm{i} 0}}{T_{\mathrm{e} 0}}\right)^{2},
$$

where $k_{y}$ is the instability wave number normalized to the inverse ion sound gyroradius $\rho_{\mathrm{s}}=$ $c_{\mathrm{s}} / \Omega_{\mathrm{i}}$, with the ion sound speed $c_{\mathrm{s}}=\left(T_{\mathrm{e} 0} / m_{\mathrm{i}}\right)^{1 / 2}$ and the ion gyrofrequency $\Omega_{\mathrm{i}}$; while $T_{j 0}$ and $m_{j}$ are the temperature and mass, respectively, with the ion and electron species indicated by the indices. The density gradient along the $x$ direction is $\omega_{n}=L_{\mathrm{ref}} / L_{n}$, utilizing the definitions $L_{\text {ref }}$ for the reference (macroscopic) normalization length and $L_{n}=-n_{0} /\left(\nabla_{x} n_{0}\right)$ for the density gradient scale length; $n_{0}$ is the background density, identical for ions and electrons due to quasineutrality. In the usual local flux tube approximation, both $\nabla_{x} n_{0}$ and $n_{0}$ are assumed to be constant throughout the simulation domain. Growth rates are normalized to $c_{\mathrm{S}} / L_{\text {ref }}$ unless indicated otherwise.

Other work on tearing modes often uses the electron diamagnetic drift frequency $\omega_{* \mathrm{e}}$ for normalization. It is straightforward to convert to that standard: in terms of the normalized density gradient $\omega_{n}$, one has $\omega_{* \mathrm{e}}=\omega_{n} k_{y} c_{s} / L_{\text {ref }}$, where $k_{y}$ is again normalized to $\rho_{\mathrm{s}}^{-1}$. Thus, this form combines the driving density gradient with the standard normalization of frequencies for this paper.

A recent study of two-dimensional reconnection in strong guide fields [5] has shown that in certain parameter regimes, Eq. (1) is no longer valid, but rather found that - under the right conditions - background gradients may enhance rather than stabilize CT modes. In that

publication, the occurrence of faster modes (relative to CT) in the presence of background 
gradients is mentioned, along with an attribution of the effect to gradient-driven instabilities.

This paper follows up on those initial findings and describes in detail the process by which background pressure gradients can enhance reconnecting field growth. It is structured as follows. After a few words on the gyrokinetic turbulence code GENE, which is used throughout this paper, it is demonstrated that Eq. (1) accurately predicts the gradientmodified growth rate in the appropriate limits and in the absence of $B_{\|}$fluctuations. While similar comparisons have been made in the past, demonstrating agreement here serves the purpose of highlighting the impact of $B_{\|}$in the later parts of this paper. A brief excursion is made in Sec. III, where a new, pressure-gradient-driven instability is presented, which relies fundamentally on $B_{\|}$. It is then shown in Sec. IV how reconnection rates may be enhanced by $\nabla B_{\|}$drifts in high- $\beta$ cases, counteracting diamagnetic drifts, as well as coupling to pressuregradient-driven instabilities. For the solar corona, the latter process is predicted to become important below a critical gradient length scale, as demonstrated in Sec. V. A summary of the results of this paper can be found in Sec. VI.

\section{CODE-THEORY COMPARISON}

As described in detail in Ref. [5] for reconnection physics, the GENE code [17, 18] solves the gyrokinetic Vlasov-Maxwell system for arbitrary species - here, ions and electrons with derivatives along the parallel coordinate treated as $\partial / \partial z \rightarrow 0$ for the present case. It is assumed that the reconnecting magnetic field $B_{x, y} \ll B_{0}$, relative to the uniform background field $B_{0}$ along $z$. Note that in the presence of $\omega_{n} \neq 0$, force balance technically requires $\partial B_{0} / \partial z \neq 0$; this effect, however, is neglected here, a common choice in local flux tubes. The perpendicular directions $x$ and $y$ are normalized to $\rho_{\mathrm{s}}$, whereas $z$ is normalized to $L_{\mathrm{ref}} \gg \rho_{\mathrm{s}}$. The gyrokinetic framework [19] orders out the gyrofrequency time scale, thus removing fast magnetosonic waves (but retaining parallel magnetic fluctuations $B_{\|}$). One may initialize the perturbed part of the distribution function such that it produces a magnetic field $B_{y 0}=B_{y}(t=0)$ at a constant $k_{x}=k_{\mathrm{cs}}$. This drives linear growth of $B_{x}$, derived from the magnetic vector potential $A_{\|}$, with $B_{x} \ll B_{y}$ throughout the linear phase of the instability. Note that all fluctuating magnetic field quantities are normalized to $B_{0} \rho_{\mathrm{s}} / L_{\text {ref }}$.

The CT mode grows in the range of wave numbers $k_{y}$ between 0 and $k_{\mathrm{cs}}-$ corresponding 




FIG. 1. Comparison of simulation data with the relative stabilization predicted by Eq. (1) for finite density gradients $\omega_{n} \equiv L_{\text {ref }} / L_{n}$. In this figure, growth rates are normalized to the inverse Alfvén time $\gamma_{\mathrm{A}}$. Good agreement is seen for these physical input parameters, which lie well in the regime of validity of the underlying theory. Simulations were run with $B_{\|}$deactivated.

to $\Delta^{\prime}$ values of $+\infty$ and 0 , respectively, see Ref. [1] - with the maximum $\gamma$ occurring roughly in the center of this $k_{y}$ region. As detailed, e.g., in Refs. [1, 5], one may write the conditions where analytical theory [16] correctly describes the linear physics of the CT mode as

$$
\begin{aligned}
& \beta\left(1+\frac{T_{\mathrm{i} 0}}{T_{\mathrm{e} 0}}\right) \gg 2 \frac{m_{\mathrm{e}}}{m_{\mathrm{i}}} \quad \text { isothermal electrons, } \\
& \beta\left(1+\frac{T_{\mathrm{i} 0}}{T_{\mathrm{e} 0}}\right) \ll 2\left(\frac{m_{\mathrm{e}}}{m_{\mathrm{i}}}\right)^{1 / 4} \quad \text { no electron FLR, no polarization drift. }
\end{aligned}
$$

Here, FLR stands for finite Larmor radius effects, and $\beta$ represents the ratio of the electron kinetic pressure to the magnetic pressure.

To comply with both conditions, the following parameter set is chosen for code-theory comparisons: $T_{\mathrm{i} 0} / T_{\mathrm{e} 0}=0.01$, hydrogen mass ratio, $\beta=0.02$, and $k_{\mathrm{cs}}=0.2$. In Fig. 1 , growth rates are shown for the $k_{y}=0.01$ tearing mode, as a function of increasing density gradient $\omega_{n}$. Equation (1) is seen to agree rather well with the simulation data; notably, the simulated growth rate at $\omega_{n}=0$ was taken for $\gamma_{0}$ to avoid sensitivity to small errors relative to the analytical small- $k_{y}$ limit. While at small values of $\beta$, the impact of $B_{\|}$tends to be very weak, it is to be stressed that these simulations were obtained while artificially setting $B_{\|}=0$. 
The growth rates shown in Fig. 1 are normalized to the inverse Alfvén time

$$
\gamma_{\mathrm{A}}=\frac{k_{\mathrm{cS}} B_{y 0, \max }}{n_{0} m_{\mathrm{i}}}\left(\frac{2}{\beta}\right)^{1 / 2},
$$

see Refs. [1, 5]. In standard GENE units, growth rates are normalized to $c_{\mathrm{s}} / L_{\mathrm{ref}}$ and depend on the strength of the reconnecting field $B_{y 0 \text {,max }}$. This standard normalization is used in the next Section, where a slab drift wave is investigated that requires no current gradient for instability.

\section{THE GRADIENT-DRIVEN DRIFT-COUPLING INSTABILITY}

In order to illuminate the enhancement of CT growth by pressure gradients for the case studied in the present paper, it is first necessary to examine a new instability. Consider a simple, unsheared slab geometry with a density gradient $\omega_{n}$ or temperature gradient $\omega_{T}$ (of any species) across the $x$ direction - here, without loss of generality, the case of only a finite $\omega_{n}$ is highlighted. The following assumptions and simplifications are made:

- no parallel variation: $\partial / \partial z \rightarrow 0$

- no radial variation: $k_{x}=0 \Rightarrow k_{\perp}=k_{y}$

- no perpendicular magnetic fluctuations: $A_{\|}=0$

- Maxwellian velocity space for the perturbed distributions

More specifically, the driftkinetic limit as applied here refers to a replacement of the Bessel functions and derived quantities in the gyrokinetic equations with first-order small-argument expansions. It should be noted that while $A_{\|}$is neglected, the coupled $\Phi-B_{\|}$system is treated self-consistently through the Poisson equation and Ampère's law.

In Appendix $\mathrm{A}$, the Vlasov equation and coupled $\Phi-B_{\|}$field equations for this case are used to derive a dispersion relation that takes the form of a quadratic equation, see Eq. (A25). Further assuming low $\beta$ and small $k_{y}$ lets one compare the relative magnitude of competing terms, significantly simplifying the dispersion relation and reducing the solution to Eq. (A31). It is further shown that, consistently using GENE conventions for normalization and frequency sign, this equation yields a growth rate and frequency that agrees very well with the corresponding output from a Maple worksheet evaluating the full dispersion 


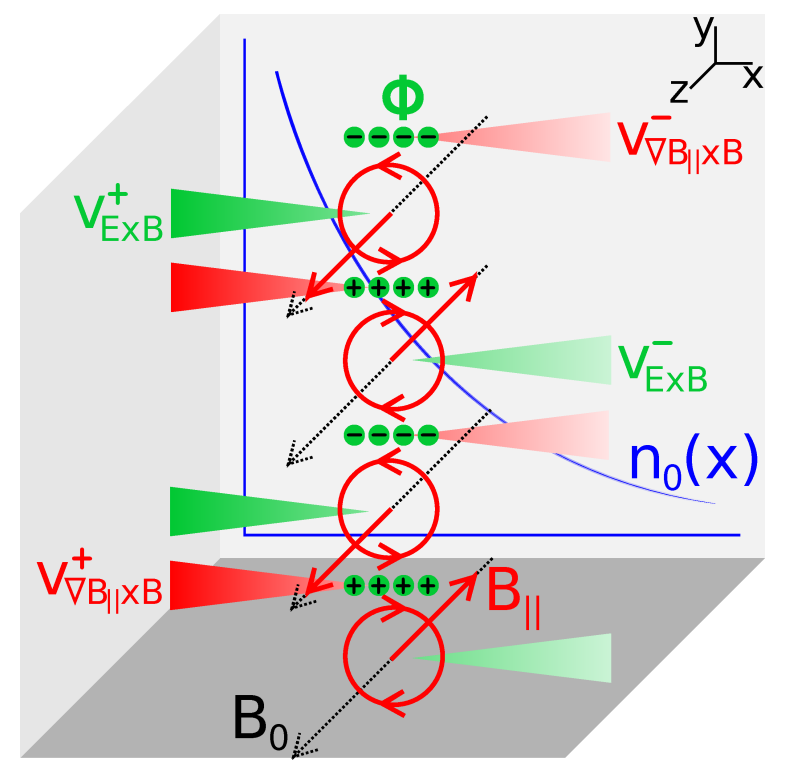

FIG. 2. (Color online) Cartoon picture of the GDC instability. Electrostatic perturbations (green charges) create drifts (green triangles), which reinforce parallel magnetic perturbations (red circles) and thus their associated drifts (red triangles). The reinforcing mechanism relies on a gradient of the background density (blue) or temperature. See the text for more details.

relation, the simulation output from GENE, and the simulation output from the gyrokinetic code AstroGK [20]. In a last step, parameter dependencies are extracted, yielding for growth rate and frequency, respectively,

$$
\gamma \propto \omega_{n} \beta^{1 / 2} \quad \text { and } \quad \omega \propto \omega_{n} k_{y}
$$

It is possible to provide a more intuitive picture of the physical mechanisms governing this instability. In Fig. 2, an electrostatic potential perturbation is shown in green in the form of alternating positive and negative charges in the $y$ direction. This $\Phi$ causes an $E \times B$ drift which advects material from regions of higher and lower background density (or temperature, in cases where a temperature gradient is present), shown here in blue. The resulting density perturbation, via pressure balance, leads to a perturbation in $B_{\|} \propto p_{\perp} \propto n T$ (red circles), where $p_{\perp}$ denotes the perturbed perpendicular pressure, at a phase angle of 90 degrees relative to the original $\Phi$ perturbation. Such a $B_{\|}$, however, necessarily causes a $\nabla B_{\|}$ drift, which, due to its charge-separating property, produces electrostatic perturbations. As this drift is phase-aligned with the original $\Phi$, reinforcement occurs, and an instability has arisen (a second solution to the equations corresponds to anti-alignment and results in a 
damped mode). Based on the above properties, this instability is hereafter referred to as the gradient-driven drift-coupling (GDC) mode. Both drift reinforcement mechanisms arise from the $\Phi-B_{\|}$cross terms in Eqs. (A21) and (A22).

The GDC can be considered a drift-wave-type instability. This is confirmed by the cross phases between the electrostatic potential $\Phi$ and density or temperatures (depending on the driving gradient), which obey the expected relations, e.g.: $\Phi \times n$ has a $+\pi / 2$ phase, $\Phi \times T_{j}$ has $-\pi / 2$ for $\omega_{n}$ drive. This mode fundamentally requires both $B_{\|}$fluctuations and background density or temperature gradients in order to grow; if $A_{\|}$is allowed to evolve self-consistently, one tends to observe $A_{\|} \lll \Phi \sim B_{\|}$. If $B_{\|}($or $\Phi)$ is suppressed in a simulation, no GDC growth is detected.

Different interpretations exist of what constitutes a drift wave. Here, no parallel electron response exists to connect density and $\Phi$ perturbations. Consequently, certain strict definitions of the term "drift wave" may not apply. However, the instability relies fundamentally on plasma drifts and also exhibits, in general, diamagnetic drifting - for most purposes, the drift wave label is therefore appropriate.

It is interesting to observe that the calculations in Appendix A also imply that the GDC is neither simply ion- or electron-type-both CT and MT are electron instabilities. Also note that modes such as MT generally require finite $A_{\|}$to reconnect fields.

Not surprisingly, the GDC grows primarily at $k_{x}=0$, as it draws on the free energy contained in the background gradient(s) along $x$. Slower growth at finite $k_{x}$ is also observed, but is found to be negligible when $k_{x} \gg k_{y}$. This can be understood by relaxing the $k_{x}=0$ assumption in the Appendix and retaining the full $k_{\perp}$. In the $z$ direction, the mode relies on the $k_{z}=0$ component: if the simulation is restricted to finite values of $k_{z}$, no growth is observed. This is simply a consequence of the fact that in the absence of any contribution at $k_{z}=0$, the mode averages out to zero along $z$.

As illustrated in Fig. 3, the parametric dependencies predicted by the solution of the dispersion relation are found to hold: as $\beta$ approaches unity (albeit only indirectly as a consequence of higher $\beta$ ), very little variation of $\gamma\left(k_{y}\right)$ can be seen for $k_{y} \ll 1$. At higher values, FLR stabilization sets in - note that even in the first-order driftkinetic limit, some FLR effects are retained, and the mode eventually becomes stable at large $k_{y}$ (larger than when using the full gyrokinetic approach). 


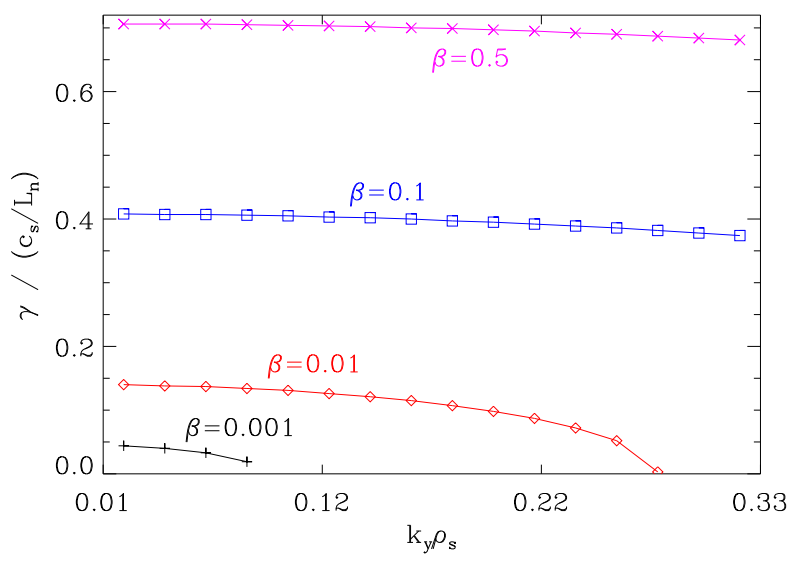

FIG. 3. (Color online) GDC growth rate spectra for different values of $\beta$ : at low pressure, the mode experiences increasing stabilization in particular at higher $k_{y}$. All results shown here use $\omega_{n}=1$ and $m_{\mathrm{i}}=m_{\mathrm{H}}$, and are normalized to $c_{\mathrm{s}} / L_{n}=c_{\mathrm{s}} / L_{\mathrm{ref}}$. The corresponding normalized diamagnetic drift frequencies therefore evaluate to $\omega_{* \mathrm{e}}=k_{y}$

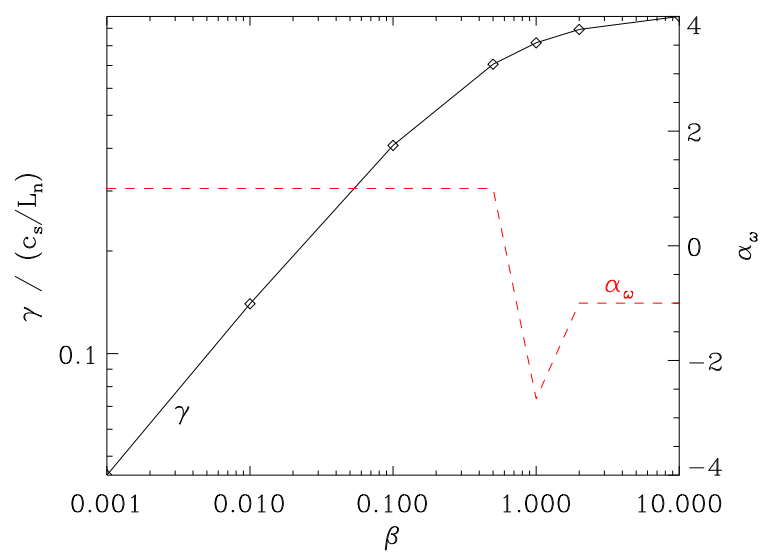

FIG. 4. (Color online) $\beta$ dependence of GDC growth (solid black line), with a different regime appearing at larger values of $\beta \gtrsim 0.1$. This is also reflected by the changing scaling of the mode frequency $\omega \propto k_{y}^{\left|\alpha_{\omega}\right|}$ (dashed red line). Negative signs of $\alpha_{\omega}$ correspond to frequencies in the electron direction. All results shown here use $\omega_{n}=1$ and $m_{\mathrm{i}}=m_{\mathrm{H}}$, and are normalized to $c_{\mathrm{s}} / L_{n}=c_{\mathrm{s}} / L_{\mathrm{ref}}$.

Figure 4 shows the influence of $\beta$ on the peak of the growth rate and on frequency scaling. Above $\beta \sim 1$, a new regime appears where the frequency changes sign from ion to electron type ( $\alpha_{\omega}$ indicates the $k_{y}$ scaling exponent of the frequency $\omega$ ), just as the low- $\beta$ scaling of $\gamma \propto \beta^{1 / 2}$ breaks down. A similar observation can be made as $T_{\mathrm{i} 0}$ is decreased relative to $T_{\mathrm{e} 0}$ 


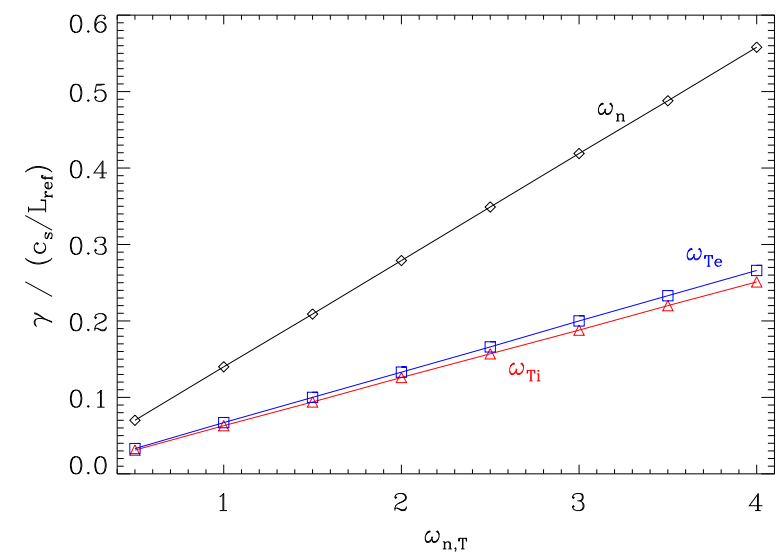

FIG. 5. (Color online) Scalings of GDC growth rate with different driving gradients: $\omega_{n}$ (black diamonds), $\omega_{T \mathrm{i}}$ (red triangles), and $\omega_{T \mathrm{e}}$ (blue squares) - for each curve, all other respective gra-


that is approximately twice as steep as the $\omega_{T j}$ curves. All results shown here use $k_{y}=0.02$ and $m_{\mathrm{i}}=m_{\mathrm{H}}$, as well as $T_{\mathrm{i} 0}=T_{\mathrm{e} 0}$.

(not shown), with $\gamma$ falling in the process.

While the derivation in Appendix $\mathrm{A}$ is based on the assumption that only a density gradient $\omega_{n}$ drives the mode, the calculation and results for finite temperature gradients $\omega_{T j}$ are almost identical. This is illustrated in Fig. 5: taking into account that the density gradient has to be applied to both ions and electrons, leading to doubled growth, the different gradients have essentially the same impact on $\gamma$. The linear dependence is in agreement with the theoretical predictions. For mixed-gradient drive, one should expect a scaling $\gamma \sim 2 \omega_{n}+\omega_{T \mathrm{i}}+\omega_{T \mathrm{e}}$. Not shown are the real frequencies - they also scale linearly with the gradients, with $[\gamma / \omega]\left(\omega_{n}\right) \approx 14,[\gamma / \omega]\left(\omega_{T \mathrm{i}}\right) \approx 2.1$, and $[\gamma / \omega]\left(\omega_{T \mathrm{e}}\right) \approx-3.3$. The sign change is to be expected for $\omega_{T \mathrm{e}}$ drive, as it signifies a transition from ion-type to electron-type mode.

As the reaction of the GDC to finite collisionality may influence its relevance to certain practical applications, its impact on the growth rate is studied next. Using the same parameters as below in Sec. IV, along with an electron-ion collisionality $\nu=0.1 c_{\mathrm{s}} / L_{\mathrm{ref}}-$ comparable in magnitude to $\gamma$ for these parameters - one finds only a minimal growth rate enhancement of $\sim 1 \%$. In effect, the GDC growth rate is thus concluded to be essentially independent of $\nu$. For more details on the Boltzmann collision operator and definition of 
the collisionality, see Ref. [5].

Studying more complex scenarios for the GDC has to be deferred to future work. However, it should be mentioned that the GDC is stabilized by the introduction of a sufficient amount of background magnetic shear; it is therefore more likely to be prevalent in low-shear configurations of magnetic confinement fusion experiments. Despite that property, a possible relation may exist with the compressional electron drift wave mentioned in Ref. [21]. There, an instability is identified which relies on pressure gradients and high $\beta$, and which exists only when taking $B_{\|}$fluctuations into account. In general, the GDC as well as its coupling to tearing modes - as detailed in the next section - could be active in magnetic confinement experiments. The impact of either, relative to other microinstabilities such as MT, would depend on the specific physical parameter regime.

In the unsheared slab, the GDC does not exhibit a critical gradient, and it is unstable - with non-negligible growth rates - even as $k_{y} \rightarrow 0$ (but not at exactly $k_{y}=0$; there, the drive term in the Vlasov equation becomes exactly zero, and no mode growth is observed). While these features make it an interesting candidate for various low-gradient astro- and space-physical applications, they also mean that nonlinear simulations of GDC turbulence are problematic. More specifically, since it is impossible to extend the simulation box in $y$ to the point where the lowest finite $k_{y}$ in the system is linearly stable, and since that very $k_{y}$ mode necessarily is the most unstable mode, turbulence levels scale with the box size, and numerical convergence in that direction is impossible. Unless additional degrees of complexity (such as magnetic curvature or a form of low- $k_{y}$ damping term) are introduced, simulations can only be considered converged if the full system size is covered in $y$. That, however, may be inconvenient due to the periodic boundary conditions. In nonlinear tests of pure GDC turbulence (i.e., without current sheet drive), the simulations were not wellbehaved. Cases with mixed GDC-CT drive tend to be somewhat more manageable, as shall be demonstrated later in this paper.

The findings of this Section shall be applied to a reconnecting system in the Section below, where the direct and indirect impact of $B_{\|}$fluctuations on reconnection is studied. 


\section{ENHANCED RECONNECTION RATES}

As described in Ref. [5], finite $\omega_{n}$ or $\omega_{T}$ in conjunction with self-consistent evolution of $B_{\|}$leads to destabilization of reconnecting field growth, generally with only moderate effects observed at higher $k_{y}$ and more severe changes at lower $k_{y}$. Before the focus is turned to the role of the GDC and similar instabilities in enhancing reconnection rates especially at these lower $k_{y}$, the direct effect of $B_{\|}$on the tearing mode shall be elucidated, which is responsible for the more moderate modifications of growth rates seen primarily at higher $k_{y}$ in the example mentioned above.

\section{A. Counteracting-Drift Destabilization}

It is of significance that the standard parameter set in Ref. [5] - which shall also be used in the present Section - espouses a large $\beta$ value of 0.3 , as it affects the relative magnitudes of drifts. The other physical parameters are $T_{\mathrm{i} 0}=T_{\mathrm{e} 0}, m_{\mathrm{e}}=0.04 m_{\mathrm{i}}, k_{\mathrm{cs}}=0.2$, and zero collisionality and dissipation. Apart from the temperature ratio, this parameter set is identical with Case II as described in Ref. [1].

For sufficiently low values of $\omega_{n}$, even relatively low $k_{y}$ tend to exhibit no unexpected behavior, i.e.: no rapid growth. At $k_{y}=0.02$, the following observations can be made. If $B_{\|}$ is switched off, increasing $\omega_{n}$ from 0 to 0.1 leads to a reduction of the linear growth rate by $0.2 \%$ - note that the growth rates were extracted with very high precision for this analysis. This is consistent with diamagnetic drift stabilization, although at these parameters, Eq. (1) can no longer be expected to yield precise quantitative predictions. Conversely, when $B_{\|}$ is included, increasing $\omega_{n}$ from 0 to 0.1 causes a $1 \%$ increase of $\gamma$. This opposite behavior can be explained through the newly activated term in the Vlasov equation: the $\nabla B_{\|}$drift requires both $B_{\|}$and a non-zero pressure gradient. Especially in cases of high $\beta$, it gains strength relative to the $E \times B$ drift and may counteract diamagnetic flow stabilization.

\section{B. Gradient-Enhanced Tearing}

The potentially more severe consequence of adding pressure gradients arises from coupling of the CT-driving current sheet to other instabilities, as shall be demonstrated below. While the present work is focused on coupling specifically with GDC, one could envision, in 

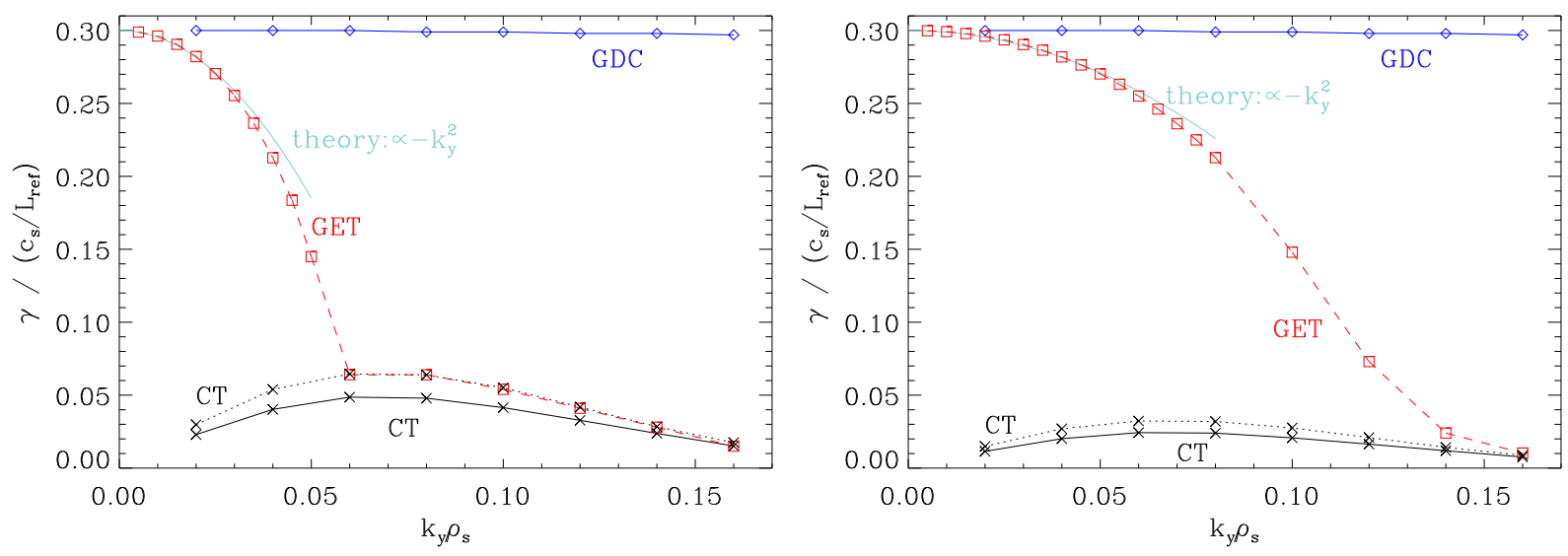

FIG. 6. (Color online) Tearing growth rates in as a function of wave number $k_{y}$ of the CT mode in absence of GDC action (black crosses) for $\omega_{n}=0$ (solid line) and extrapolated to $\omega_{n}=0.5$ (dotted line), see the text. The CT in the left plot has stronger tearing drive $B_{y 0 \text {,max }}=3.233$ than that in the right (1.617). The other data shown (all with $\omega_{n}=0.5$ ) corresponds to GDC growth in $\Phi$ and $B_{\|}$(blue diamonds) and the GET effect (red squares), see the text. As a cyan solid line, a fit curve is shown to illustrate the $k_{y}^{2}$ dependence of GET. The effect of changing CT drive relative to $\omega_{n}$ drive on GET is apparent when comparing both plots. The normalized diamagnetic drift frequencies are $0.5 k_{y}$ for either case, whereas the inverse Alfvén times $\gamma_{\mathrm{A}}$ evaluate to 1.67 (left) and 0.84 (right).

principle, other drift-wave-type instabilities taking its place.

A clarifying comment shall be made before presenting any results, however. The terms tearing and reconnection are used for a variety of physical effects and scenarios, with different meanings found in different publications. In the present work, both refer - interchangeably to growth in the reconnecting field $B_{x}$ and the resulting rearrangement of magnetic topology around $\mathrm{X}$ and $\mathrm{O}$ points. Thus, tearing includes but is clearly not limited to the tearing mode (CT in the present nomenclature), and tearing allows no inferences about the free energy source of reconnection.

Figure 6 shows the CT growth rate in black, both for $\omega_{n}=0$ and $\omega_{n}=0.5$, with the latter extrapolated to illustrate the subdominant regime at low $k_{y}$. For the extrapolation, simulations with lower $\omega_{n}$ are used, and the moderate enhancement of $\gamma$ (as opposed to the rapid growth discussed below) seen in these simulations - which varies little over the entire $k_{y}$ range - is used to obtain $\gamma$ at $\omega_{n}=0.5$. The resulting values match the high- $k_{y}$ direct 


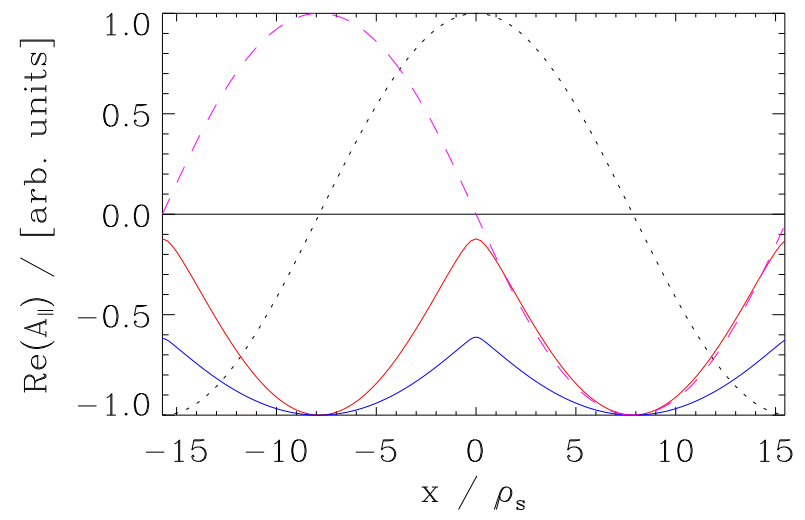

FIG. 7. (Color online) Radial mode structures $\operatorname{Re}\left(A_{\|}\right)$, in arbitrary units, for CT (solid lines: red for $k_{y}=0.2$, blue for $k_{y}=0.16$ ), for the driving current sheet (black dotted line), and for GET (pink dashed line, $k_{y}=0.22$ ). Here, a particularly small $B_{y 0 \text {,max }}$ was chosen to obtain GET growth at negative $\Delta^{\prime}$.

results at this gradient setting very precisely. Also included, in red, is the enhanced growth rate of the process which henceforth will be referred to as gradient-enhanced tearing (GET). Growth rates for CT and GET are extracted by measuring the reconnecting field $B_{x}$ as a function of time - since the GDC does not excite $B_{x, y}$ fluctuations, this does not measure linear GDC growth (which instead is apparent in $\Phi$ and $B_{\|}$).

In Fig. 6, the blue curve labeled GDC marks growth of the linear system in the absence of a current gradient. In terms of numerical resolutions, the GDC requirements are similar to those for CT (see Ref. [5]), apart from the radial direction which technically requires only one mode at $k_{x}=0$-compare, however, the corresponding comment in the previous Section.

The effect of $\nabla B_{\|}$on CT growth rates, as discussed earlier, can be seen by comparing the solid black curve (CT at $\omega_{n}=0$ ) and the dotted black curve (CT at $\omega_{n}=0.5$ ). A moderate enhancement is found to exist throughout the range of unstable $k_{y}$.

The picture becomes more complex when looking at the GET simulation results at $\omega_{n}=$ 0.5, as shown by the dashed red curves: while following the dotted black curve at high $k_{y}$, growth rates much larger than that of the $\mathrm{CT}$ are observed at low $k_{y}$. As $k_{y} \rightarrow 0$, the growth rate of the GDC is attained (in units of $c_{\mathrm{S}} / L_{\mathrm{ref}}$ ). The deviation from the original $\mathrm{CT}$ behavior becomes even more striking when lowering the driving $B_{y 0 \text {,max }}$, as seen in the right plot. Here, the growth rates especially at low $k_{y}$ take on more and more the nature 


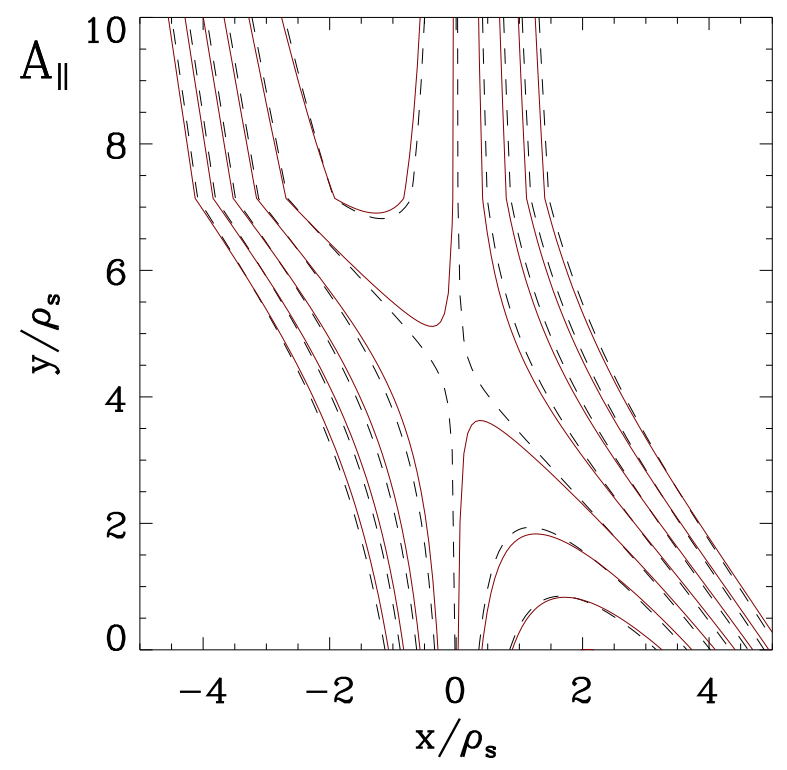

FIG. 8. (Color online) Contours of the magnetic potential $A_{\|}$for two narrowly spaced moments in time: earlier contours are black dashed lines, later ones red solid lines. Only a subsection of the simulation box is shown, focusing on the $\mathrm{X}$ point, where reconnection is clearly visible. The data stems from a GET simulation at negative $\Delta^{\prime}$ with a large $\omega_{n}$ relative to the tearing mode drive.

of the GDC rather than the CT mode. One consequence is that CT growth occurs even for $k_{y} \geq k_{\mathrm{cs}}$ if the GDC is sufficiently strong (not shown in the figure), despite the fact that this corresponds to the region of $\Delta^{\prime} \leq 0$. The relevant mode structures are shown in Fig. 7, including GET for one such case $\left(k_{y}=0.22\right)$. Note that due to the dominant $k_{x}=0$, the GDC structure (not shown) is constant in $x$. Due to its coupling to the current sheet, GET results in a corresponding mode structure when CT is subdominant. As soon as the red GET curve becomes identical with the black CT curve in Fig. 6, its structure matches exactly that of the CT.

In light of the mode parities in Fig. 7, it is to be stressed that GET, while not a tearing mode in the strict sense of the word, is indeed able to reconnect magnetic fields at an elevated rate. This is illustrated in Fig. 8, where reconnection at the rate of the GDC occurs at $k_{y}=0.22>k_{\mathrm{cs}}$ (i.e., $\left.\Delta^{\prime}<0\right)$, as a consequence of a large $\omega_{n}$.

Destabilization of reconnection by GDC action at lower $k_{y}$ can be understood from a heuristic calculation. CT simulations - here, in Ref. [5], and equivalently in Ref. [1] - are technically nonlinear but yield linear growth rates and describe linear physics. This stems 
from the fact that the current sheet implementation occurs purely in the perturbed modified distribution function $g(k)$ through the Vlasov nonlinearity $\sum_{k^{\prime}}\left(k_{x}^{\prime} k_{y}-k_{x} k_{y}^{\prime}\right) \chi\left(k^{\prime}\right) g\left(k-k^{\prime}\right)$, with $\chi$ containing the current sheet (rather than a perturbation thereof).

Here, the effect on the linear growth rate is of interest. It is a mode-coupling term in the gyrokinetic response for $g(k, \omega)$ that is proportional to $g\left(k-k^{\prime}, \omega-\omega^{\prime}\right)$, nominally making it phase-incoherent with $g(k, \omega)$. One may use the renormalization procedure of resonance broadening theories [22] to calculate iterative mode-coupling contributions in order to find the piece that is phase-coherent with $g(k, \omega)$. To model the effect on the growth rate, the frequency of the gyrokinetic operator is taken here to represent the frequency of the linear dispersion relation - which is not be modeled explicitly in the following - and find the nonlinear decrement from the renormalized nonlinearity.

The nonlinear gyrokinetic response of the GET effect at wavenumber $k$ and frequency $\omega$ can be written as

$$
-i \omega g(k, \omega)=\sum_{k^{\prime}, \omega^{\prime}}\left(k_{x}^{\prime} k_{y}-k_{x} k_{y}^{\prime}\right) \chi\left(k^{\prime}, \omega^{\prime}\right) g\left(k-k^{\prime}, \omega-\omega^{\prime}\right) .
$$

GET is observed for the first component in the nonlinearly interacting triplet of $(k, \omega)$, $\left(k^{\prime}, \omega^{\prime}\right)$, and $\left(k-k^{\prime}, \omega-\omega^{\prime}\right)$ with a complex frequency $\omega$ to be determined from the renormalized dispersion relation to be derived here. The second triplet component is the current sheet that drives the $\mathrm{CT}$, which resides in $A_{\|}$, and therefore in $\chi$ at $k_{x}^{\prime}=k_{\mathrm{cs}}$ and $k_{y}^{\prime}=0$. It is stationary (whereby the nonlinearity physically reduces to a linear term, albeit one with mode coupling), converting the frequency summation to the operation $\omega^{\prime} \rightarrow 0$. Lastly, the GDC is the third component in the triplet, with $k_{x}-k_{x}^{\prime}=0$ (which makes $k_{x}=k_{\mathrm{cs}}$ ) and $k_{y}-k_{y}^{\prime}=k_{y}>0$. The current sheet and GDC act as the dominant terms in the $k^{\prime}$ summation of the Vlasov nonlinearity, with the generalized potential $\chi\left(k^{\prime}\right)$ reducing to $-v_{\|} A_{\|}\left(k_{\mathrm{cs}}, 0\right)$ (ignoring gyroaverages), with $v_{\|}$denoting the parallel velocity coordinate.

Determining the coherent renormalization of the right-hand side of Eq. (6) is done by iterating on the mode coupling to find a nonlinear component that is phase-coherent with $g(k)$. The (technically nonlinear) evolution of $g\left(k-k^{\prime}, \omega-\omega^{\prime}\right)=g\left(k-k^{\prime}, \omega\right)$ is expressed in terms of its linear GDC drive and its mode coupling as follows,

$$
-i\left(\omega-\omega_{\mathrm{GDC}}\right) g\left(k-k^{\prime}, \omega\right)=\sum_{k^{\prime \prime \prime}}\left(k_{x}^{\prime \prime \prime}\left(k_{y}-k_{y}^{\prime}\right)-\left(k_{x}-k_{x}^{\prime}\right) k_{y}^{\prime \prime \prime}\right) \chi\left(k^{\prime \prime \prime}\right) g\left(k-k^{\prime}-k^{\prime \prime \prime}, \omega\right),
$$

where the appearance of the complex GDC frequency leads to a linear response $\omega=\omega_{\text {GDC }}$ 
for $\chi=0$, consistent with GDC wavenumber $k-k^{\prime}$. The wavenumber $k^{\prime \prime \prime}=-k^{\prime}$ selects the coherent renormalized response. Solving this expression for $g\left(k-k^{\prime}, \omega\right)$ and substituting it into Eq. (6) yields

$$
-i \omega g(k, \omega)=-\frac{\sum_{k^{\prime}}\left(k_{x}^{\prime} k_{y}-k_{x} k_{y}^{\prime}\right)^{2}\left|\chi\left(k^{\prime}\right)\right|^{2}}{-i\left(\omega-\omega_{\mathrm{GDC}}\right)} g(k, \omega),
$$

where the right-hand side is a standard propagator renormalization or resonance broadening decrement (see, e.g., Ref. [23]). The solution of the resulting dispersion relation is

$$
\omega=\frac{1}{2}\left[\omega_{\mathrm{GDC}} \pm\left(\omega_{\mathrm{GDC}}^{2}+4 \sum_{k^{\prime}}\left(k_{x}^{\prime} k_{y}-k_{x} k_{y}^{\prime}\right)^{2}\left|\chi\left(k^{\prime}\right)\right|^{2}\right)^{1 / 2}\right] \equiv \omega_{\mathrm{GET}}
$$

whose dominant branch reduces to $\omega_{\mathrm{GET}}=\omega_{\mathrm{GDC}}$ for a negligible current sheet in $\chi$.

Assuming a weak but non-zero current sheet, the first and (at least at low $k_{y}$ ) dominant root of the dispersion relation becomes

$$
\omega_{\mathrm{GET}}=\omega_{\mathrm{GDC}}\left(1+\frac{1}{\omega_{\mathrm{GDC}}^{2}} \sum_{k^{\prime}}\left(k_{x}^{\prime} k_{y}-k_{x} k_{y}^{\prime}\right)^{2}\left|\chi\left(k^{\prime}\right)\right|^{2}\right),
$$

which, for $\omega_{\mathrm{GDC}}=i \gamma_{\mathrm{GDC}}$, results in overall growth that experiences a stabilizing force at finite $k_{y}$ that is proportional to $k_{y}^{2} B_{y 0 \text {,max }}^{2}$, which is precisely what was shown to be the case in Fig. 6.

More specifically, quadratic fits to the data at low $k_{y}$ are shown for both plots. One may compare these fits quantitatively with the predicted values from Eq. (10), using for this simple argument the thermal velocity (i.e., $v_{\|} \sim 1$ in normalized units) in the definition of $\chi$, as well as $k_{\mathrm{cs}} A_{\|} \sim B_{y 0 \text {,max }}$. For the first case, $B_{y 0 \text {,max }}=3.233$, one thus obtains a stabilization $\Delta \gamma$ about $25 \%$ weaker than the fit curve. Repeating this procedure for the second case, $B_{y 0, \max }=1.617$, very similarly yields a $\Delta \gamma$ about $25 \%$ lower than the corresponding fit curve. Therefore, despite the heuristic nature of the calculation, it is able to capture the dependencies on $k_{y}$ and $B_{y 0, \max }$ correctly, as well as provide a fairly good quantitative estimate for the stabilization.

Having established the physical process behind GET, the focus is now shifted to the regulation of either growth rate through the driving gradients. CT growth scales with $\gamma_{\mathrm{A}} \propto B_{y 0, \max }$, whereas in units of $c_{\mathrm{s}} / L_{\mathrm{ref}}$, the value of $\gamma_{\mathrm{GDC}}$ depends only on $\omega_{n}\left(\right.$ if $\left.\omega_{T j}=0\right)$ and yields results independent of $B_{y 0, \max }$ (when outside the GET regime). Note that $L_{\mathrm{ref}}$ enters both into the normalization of the reconnecting field via $B_{y 0 \text {,max }} \rightarrow B_{y 0 \text {,max }} B_{0} \rho_{\mathrm{s}} / L_{\mathrm{ref}}$ 
and the GDC growth rate measured in units of $c_{\mathrm{s}} / L_{\text {ref. }}$. Consequently, $L_{\text {ref }}$ can be seen as a measure of the scale separation between reconnecting and background field, while simultaneously determining the value of $\omega_{n}$ for a given $L_{n}$.

Consider a hydrogen plasma in a uniform magnetic field $B_{0}$ which is subjected to a (perpendicular) density gradient $\omega_{n}=1$, equivalent to normalizing macroscopic scales to the gradient scale length. For simplicity, assume $\beta=2$ and $n_{0}=T_{\mathrm{i} 0}=T_{\mathrm{e} 0}=1$. Now a current is introduced in the parallel direction which produces a sinusoidal field $B_{y}$ at $k_{x}=1$ which is constant in $y$. This scenario is susceptible to a CT mode with a growth rate maximum of $\gamma_{\mathrm{CT}} \sim 0.03 \gamma_{\mathrm{A}}$; at the same time, it provides the ingredients for GDC/GET, this time with a growth rate on the order of $\gamma_{\mathrm{GET}} \sim c_{\mathrm{S}} / L_{n}\left(=k_{y} \omega_{* \mathrm{e}}\right.$, even though the results are insensitive to the precise value of $k_{y} \rightarrow 0$ ). Translating this value into Alfvénic units yields $\gamma_{\mathrm{GET}} \sim \gamma_{\mathrm{A}} / B_{y 0 \text {,max }}$, making the relative $\mathrm{CT}$-GET impact

$$
\frac{\gamma_{\mathrm{CT}}}{\gamma_{\mathrm{GET}}} \sim 0.03 B_{y 0, \max }
$$

where the usual normalization for $B_{y 0 \text {,max }}$ evaluates to units of $B_{0} \rho_{\mathrm{s}} / L_{n}$. By choosing a value for the free parameter $B_{y 0, \max }$, one can pick the dominant instability; in the process possibly creating a GET growth rate $\gamma_{\mathrm{GET}} \approx \gamma_{\mathrm{GDC}} \gg \gamma_{\mathrm{CT}}$, along with $\gamma_{\mathrm{GET}} \gg \gamma_{\mathrm{A}}$. Alternatively, one may view $B_{y 0 \text {,max }}$ as fixed and vary $\omega_{n}$, with the same relative results. By dint of these relations, GET may therefore take the role of ultrafast reconnection - as opposed to the CT mode which is sometimes referred to as fast reconnection.

\section{Impact On Turbulence}

Nonlinear particle acceleration through parallel electric fields may provide large energies even when $B_{y 0 \text {,max }}$ and $B_{0}$ are significantly separated. This situation is investigated in Ref. [24], where the focus lies on reconnection turbulence driven by a term in the Vlasov equation

$$
\frac{\partial g}{\partial t}=\ldots-\omega_{\mathrm{dr}}\left(g_{k y=0}(t)-g_{k y=0}(t=0)\right)
$$

where $\omega_{\mathrm{dr}}$ is a driving frequency; dissipation is provided by a collision operator. Note that this form of the nonlinear drive constitutes an improved version of that presented in Ref. [5]. Before applying GET to a fully nonlinear scenario, it should be noted that the turbulent parallel electric field $E_{\|}$- as well as the heating rate $j_{\|} E_{\|}$(with the parallel current $\left.j_{\|}\right)-$ 
scales to a significant degree in accordance with expectations based on the linear growth rates. While Ref. [24] focuses on a case without background gradients, for the present work simulations were performed at $\omega_{n}$ ranging from 0.1 to 1 . Even when linear growth rate enhancements were small (i.e., $\gamma \gtrsim \gamma\left(\omega_{n}=0\right)$ ), parallel electric fields and heating rates were significantly increased. As was discussed in Sec. III, box size settings - which, in numerically converged regions, have no impact on volumetric heating rates in the absence of $\omega_{n}-$ severely affect $j_{\|} E_{\|}$even at $\omega_{n}=0.1$.

A brief comment on the heating rate and the parallel electric field: consistent with gyrokinetic literature, the moniker parallel is used for a number of quantities that are parallel to the background magnetic field $-v_{\|}, A_{\|}, B_{\|}, j_{\|}$- and could equivalently be labeled with the subscript $z$. Magnetic field perturbations will lead to a slight misalignment of field lines relative to the background field, but it can be shown through ordering relations that the aforementioned quantities are hardly affected; e.g., $v_{\|}=v_{z}+\delta$, where $\delta \rightarrow 0$ in gyrokinetic ordering. This, however, is not the case for $E_{\|}$, where this misalignment has to be taken into account, see Ref. [24].

For the latter case, with the default box sizes from Ref. [24] of $62.8 \rho_{\mathrm{s}} \times 62.8 \rho_{\mathrm{s}}$, contours of the turbulence are shown in Fig. 9. The tendency of turbulent structures to cover the whole simulation box is apparent not only in $\Phi$ (where the GDC exerts direct influence) but also in $A_{\|}$, where GET imprints CT with GDC features. In particular, $B_{\|}$(not shown in the figure) and $\Phi$ - whose field equation is coupled to that of $B_{\|}$- see marked increases in amplitude relative to the case with $\omega_{n}=0$, with strong heat and particle transport along $x$. Interpreting the results of such a simulation in the context of physical heating therefore becomes a difficult undertaking. The addition of GDC-stabilizing properties to the physical setup, such as background magnetic shear or curvature, may alleviate these issues; due to the associated complexity, such studies will have to be deferred to future work.

While more research into the physical and numerical properties of this mixed reconnection and drift-wave turbulence will be necessary to understand all relevant mechanisms, these first results indicate that GDC and GET do not merely affect linear growth rates but can also be expected to enhance heating in turbulent scenarios. Given the difficulties and open questions that stem from nonlinear simulations, however, in the following the focus is returned to linear enhancement of reconnection rates and their possible role in coronal settings. 


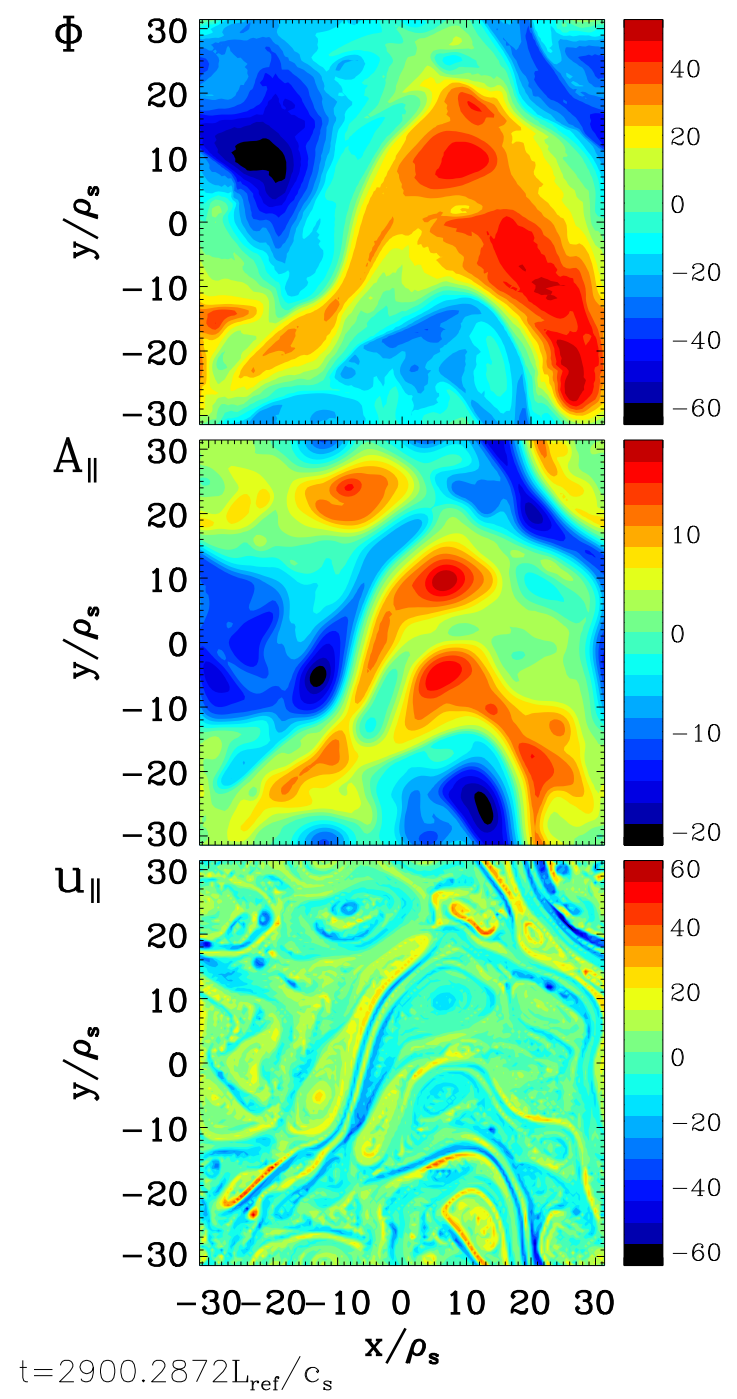

FIG. 9. (Color online) Turbulent contours, from top to bottom, of the electrostatic potential $\Phi$, the magnetic potential $A_{\|}$, and the parallel electron flow velocity $u_{\|}$(which serves as a good proxy for $j_{\|}$), for a simulation at finite $\omega_{n}=0.1$. The snapshot shown here is characteristic of the quasi-stationary turbulent state.

\section{APPLICATION TO THE SOLAR CORONA}

To gauge whether the GET process may contribute to energetic particle production in the solar corona - generally considered to be a region where magnetic reconnection is an important source of non-thermal particles - the critical (density or temperature) gradient shall now be calculated at which GET (taken here to be driven by a GDC mode) overtakes standard CT as the dominant process. 
As per Refs. [25, 26], typical parameters for the solar corona are $n \sim 10^{9} \mathrm{~cm}^{-3}$ for the density, $B_{0} \sim 100 \mathrm{G}$ for the magnetic field, and $L \sim 10^{9} \mathrm{~cm}$ for the parallel extent of the domain and choice of $L_{\text {ref }}$; with a temperature $T=10^{6} \mathrm{~K}$ which shall be applied here to both ions and electrons. From these quantities, one arrives at $\beta=0.00035, c_{\mathrm{s}}=9.1 \times 10^{6} \mathrm{~cm} / \mathrm{s}$, and $\rho_{\mathrm{s}}=9.5 \mathrm{~cm}$. These values are identical with the default settings used in the Corona section of Ref. [24].

Furthermore, using the respective domain size and magnetic field from Ref. [25], one has a driving $k_{x}=0.0004$ and field $B_{y 0, \max }=7.5 \times 10^{6}$ in the usual normalized units (corresponding to $B_{y 0}=2^{-1 / 2} B_{y 0, \max }=5 \mathrm{G}$ ). Simulating at lower $B_{y 0, \max }$ and using the standard $\gamma_{\mathrm{A}}$ scaling, one can extract the $\mathrm{CT}$ growth rate; the corresponding simulations were performed with $N_{x}=16384$ grid points in $x$. In addition, one can directly obtain $\gamma_{\mathrm{GDC}}\left(\omega_{n}\right) \approx \gamma_{\mathrm{GET}}\left(\omega_{n}\right)$ (with similar results expected for $\omega_{T j}$ which are set to zero here) for these parameters.

In units of $c_{\mathrm{s}} / L_{\mathrm{ref}}$, the growth rates thus read: $\gamma_{\mathrm{CT}} \sim 1.3$ and $\gamma_{\mathrm{GET}}=0.026 \omega_{n}$; or, equivalently, $\gamma_{\mathrm{CT}} / \gamma_{\mathrm{A}} \sim 5.7 \times 10^{-6}$ and $\gamma_{\mathrm{GET}} / \omega_{* \mathrm{e}}=65$ (assuming $k_{y}=k_{\mathrm{cs}}$ in the definition of the diamagnetic drift frequency). Based on these values, the critical gradient length scale where $\gamma_{\mathrm{CT}}=\gamma_{\mathrm{GET}}$ becomes

$$
L_{p}^{\text {crit }}=0.02 L_{\text {ref }}=2 \times 10^{7} \mathrm{~cm}
$$

where $L_{p}=L_{n}$ or $L_{p}=L_{T}$. Clearly, this value scales with $B_{y 0, \max }$ : smaller values will result in an earlier onset of GET - an alternative interpretation is that for a given $\omega_{n, T}$, there exists a minimal $B_{y 0 \text {,max }}$ below which GET will dominate. If gradients occur on smaller scale lengths than $L_{p}^{\text {crit }}$, and presuming that three-dimensional CT is not drastically faster than its two-dimensional counterpart, one can expect GET to play a role in reconnecting fields. It should be stressed that $L_{p} \gg L_{p}^{\text {crit }}$ may be necessary for GET to be straightforward to distinguish from CT observationally.

While large-scale density or temperature fluctuations in the corona are on the order of $L_{p} \sim 10^{8} \mathrm{~cm}$, filamentation can create significantly smaller-scale fluctuations [27] with potentially higher $\omega_{n, T}$. In particular, the above $L_{p}^{\text {crit }}$ should constitute a realistic scenario for corona physics, making GET activity an interesting candidate for processes behind coronal heating. Moreover, the filament-like structures in the turbulence described in Ref. [24] are creating small-scale, self-consistent gradients in $n$ and $T$, while mergers of circular structures 
are found to create heating bursts consistent with nanoflares [28]. Therefore, one may assume that nanoflare intensities can be affected by the GET effect.

\section{SUMMARY}

Gradients in the background density and their impact on tearing mode growth rates have been studied, in conjunction with the investigation of a new drift-wave instability referred to as gradient-driven drift-coupling (GDC) mode.

The standard picture of reconnection stabilized by diamagnetic flows in response to such gradients is confirmed quantitatively in the applicable limits. However, when considering higher $\beta$ values and including $B_{\|}$effects, the $\nabla B_{\|}$drift is found to counteract the $E \times B$ drift, under the right circumstances leading to net destabilization of the CT mode. For all cases studied here, however, destabilization has been moderate, with at most a few $10 \%$ increase in growth rates.

With both $B_{\|}$and density or temperature gradients $\omega_{n, T}$, a drift-wave-type mode becomes unstable which couples the $\nabla B_{\|}$and $E \times B$ drifts. More specifically, a fluctuation in the electrostatic potential $\Phi$ reinforces, via its associated $E \times B$ drift and the background gradient $\omega_{n, T}$, the fluctuation in $B_{\|}$. The latter, in turn, reinforces $\Phi$ via the $\nabla B_{\|}$drift. Therefore, the name gradient-driven drift-coupling instability is introduced for this mode. In unsheared slab geometry, it has no critical gradient and is independent of the mode number $k_{y}$ at sufficiently low $k_{y}$.

When both CT and GDC activity are present in a system, the latter may couple to the current sheet driving the former, leading to reconnection being observed on time scales associated with GDC growth. As the GDC is regulated by $\omega_{n, T}-$ as opposed to the reconnecting field strength $B_{y 0 \text {,max }}$, which governs CT growth - there now exists a means of inducing reconnecting field growth, in principle, at a rate much faster than the inverse Alfvén time; or, alternatively, at a velocity much faster than the Alfvén speed. For this process, dubbed gradient-enhanced tearing, or GET, to be dominant over standard CT growth in the solar corona, a sufficiently large $\omega_{n, T}$ is required. Using typical parameters, the corresponding

critical gradient scale length is shown to be $L_{p}^{\text {crit }}=2 \times 10^{7} \mathrm{~cm}$, below which the GDC and thus GET grow more quickly than CT - a value that is consistent with expectations for the solar corona. 
In terms of turbulence, the impact of GET is significant: heating rates are found to be strongly enhanced even at moderate $\omega_{n}$. However, the increases in $j_{\|} E_{\|}-$and turbulent amplitudes in general - are not and cannot be made to be converged in the perpendicular box sizes, as turbulent structures are gravitating towards the lowest wave numbers in the system. As even lower wave numbers are included by increasing box sizes, the turbulence therefore changes quantitatively, leading to stronger heating among other consequences. To obtain properly converged answers regarding the impact of pressure gradients on reconnection turbulence, one must therefore either take the full system size into account - with some consequences for the boundary conditions - or introduce more realistic modeling of stabilizing effects such as background magnetic shear. Such efforts shall be the subject of future investigation.

\section{ACKNOWLEDGMENTS}

The authors would like to express their gratitude to E.G. Zweibel, V.V. Mirnov, M. Swisdak, J.F. Drake, and M. Velli for discussions and helpful insights. In part, the numerical studies in this work were performed on the Kraken XT5 HPC system through XSEDE grant PHY130013. The research leading to these results has received funding from the European Research Council under the European Union's Seventh Framework Programme (FP7/20072013)/ERC Grant Agreement No. 277870.

\section{Appendix A: Derivation}

Following Ref. [5] and the simplifications delineated in Sec. II, the normalized gyrokinetic Vlasov equation (note that $\partial_{t} \rightarrow i \omega$ instead of $-i \omega$ is used to match GENE's frequency sign convention) reads

$$
i \omega g_{j}=-\omega_{n} F_{0} i k_{y} \chi=-\omega_{n} F_{0} i k_{y}\left(J_{0}\left(\lambda_{j}\right) \Phi+\frac{T_{j 0} \mu}{q_{j}} \frac{2 J_{1}\left(\lambda_{j}\right)}{\lambda_{j}} B_{\|}\right)
$$

with $\lambda_{j} \equiv k_{\perp}\left(2 T_{j 0} m_{j} \mu /\left(B_{0} q_{j}^{2}\right)\right)^{1 / 2}$ and $\chi=J_{0}\left(\lambda_{j}\right) \Phi+\left[\left(T_{j 0} \mu\right) / q_{j}\right]\left[\left(2 J_{1}\left(\lambda_{j}\right)\right) / \lambda_{j}\right] B_{\|}$in the absence of $A_{\|}$-type fluctuations. Defining $\varpi \equiv-\omega_{n} k_{y} / \omega$, one thus obtains

$$
g_{j}=\varpi F_{0}\left(J_{0}\left(\lambda_{j}\right) \Phi+\frac{T_{j 0} \mu}{q_{j}} \frac{2 J_{1}\left(\lambda_{j}\right)}{\lambda_{j}} B_{\|}\right) .
$$


Additionally, the background distribution can be written as $F_{0} \equiv \pi^{-3 / 2} \mathrm{e}^{-v_{\|}^{2}-\mu B_{0}}$. Next, the field equations (setting the Debye length $\lambda_{\mathrm{D}}=0$ ) can be written as

$$
\begin{gathered}
\Phi=\frac{\mathcal{C}_{3} \mathcal{M}_{00}-\mathcal{C}_{2} \mathcal{M}_{01}}{\mathcal{C}_{1} \mathcal{C}_{3}-\mathcal{C}_{2}^{2}} \\
B_{\|}=\frac{\mathcal{C}_{1} \mathcal{M}_{01}-\mathcal{C}_{2} \mathcal{M}_{00}}{\mathcal{C}_{1} \mathcal{C}_{3}-\mathcal{C}_{2}^{2}} \\
\mathcal{M}_{00}=\sum_{j} q_{j} n_{j 0} \pi B_{0} \int J_{0} g_{j} \mathrm{~d} v_{\|} \mathrm{d} \mu \\
\mathcal{M}_{01}=\sum_{j} q_{j} n_{j 0} \pi B_{0}^{3 / 2} \frac{v_{T j}}{k_{\perp}} \int \mu^{1 / 2} J_{1} g_{j} \mathrm{~d} v_{\|} \mathrm{d} \mu \\
\mathcal{C}_{1}=k_{\perp}^{2} \lambda_{D}^{2}+\sum_{j} \frac{q_{j}^{2} n_{j 0}}{T_{j 0}}\left(1-\Gamma_{0}\right) \\
\mathcal{C}_{2}=-\sum_{j} \frac{q_{j} n_{j 0}}{B_{0}}\left(\Gamma_{0}-\Gamma_{1}\right) \\
\mathcal{C}_{3}=-\frac{2}{\beta}-\sum_{j} \frac{2 n_{j 0} T_{j 0}}{B_{0}^{2}}\left(\Gamma_{0}-\Gamma_{1}\right)
\end{gathered}
$$

where $\Gamma_{k} \equiv I_{k}\left(b_{j}\right) \mathrm{e}^{-b_{j}}$ and $b_{j} \equiv k_{\perp}^{2} T_{j 0} m_{j} /\left(q_{j}^{2} B_{0}^{2}\right)$. One may define

$$
\mathcal{D} \equiv\left(\mathcal{C}_{1} \mathcal{C}_{3}-\mathcal{C}_{2}^{2}\right)^{-1}
$$

Next, $g_{j}$ will be inserted into $\mathcal{M}_{0 x}$, which in turn goes into the fields.

First, these are the relevant integrals, with $\eta \sqrt{\mu} \equiv \lambda_{j}$ :

$$
\begin{aligned}
\int_{-\infty}^{\infty} \mathrm{e}^{-v_{\|}^{2}} \mathrm{~d} v_{\|} & =\pi^{1 / 2} \\
\int_{0}^{\infty} J_{0}(\eta \sqrt{\mu})^{2} \mathrm{e}^{-\mu B_{0}} \mathrm{~d} \mu & =\frac{1}{B_{0}} \mathrm{e}^{-\eta^{2} /\left(2 B_{0}\right)} I_{0}\left(\frac{\eta^{2}}{2 B_{0}}\right) \equiv \frac{\Gamma_{0}}{B_{0}} \\
\int_{0}^{\infty} J_{0}(\eta \sqrt{\mu}) J_{1}(\eta \sqrt{\mu}) \mu^{1 / 2} \mathrm{e}^{-\mu B_{0}} \mathrm{~d} \mu & =\frac{\eta}{2 B_{0}^{2}}\left(\Gamma_{0}-\Gamma_{1}\right) \\
\int_{0}^{\infty} J_{1}(\eta \sqrt{\mu})^{2} \mu \mathrm{e}^{-\mu B_{0}} \mathrm{~d} \mu & =\frac{\eta^{2}}{2 B_{0}^{3}}\left(\Gamma_{0}-\Gamma_{1}\right)
\end{aligned}
$$

Thus,

$$
\begin{aligned}
& \mathcal{M}_{00}=\sum_{j} q_{j} n_{j 0} \varpi\left(\Gamma_{0} \Phi+\frac{2 T_{j 0}}{q_{j}} \frac{1}{2 B_{0}}\left(\Gamma_{0}-\Gamma_{1}\right) B_{\|}\right) \\
& \mathcal{M}_{01}=\sum_{j} q_{j} n_{j 0} B_{0}^{1 / 2} \frac{v_{T j}}{k_{\perp}} \varpi\left(\frac{\eta}{2 B_{0}}\left(\Gamma_{0}-\Gamma_{1}\right) \Phi+\frac{2 T_{j 0}}{q_{j}} \frac{\eta}{2 B_{0}^{2}}\left(\Gamma_{0}-\Gamma_{1}\right) B_{\|}\right)
\end{aligned}
$$


These reduce to

$$
\begin{aligned}
& \mathcal{M}_{00}=\Phi \varpi \sum_{j} q_{j} n_{j 0} \Gamma_{0}+B_{\|} \varpi \sum_{j} \frac{n_{j 0} T_{j 0}}{B_{0}}\left(\Gamma_{0}-\Gamma_{1}\right) \\
& \mathcal{M}_{01}=\Phi \varpi \sum_{j} \frac{n_{j 0} T_{j 0}}{B_{0}}\left(\Gamma_{0}-\Gamma_{1}\right)+B_{\|} \varpi \sum_{j} \frac{2 n_{j 0} T_{j 0}^{2}}{q_{j} B_{0}^{2}}\left(\Gamma_{0}-\Gamma_{1}\right)
\end{aligned}
$$

or, implicitly defining the quantities $\mathcal{A}$,

$$
\begin{aligned}
& \mathcal{M}_{00}=\Phi \varpi \mathcal{A}_{1}+B_{\|} \varpi \mathcal{A}_{2} \\
& \mathcal{M}_{01}=\Phi \varpi \mathcal{A}_{2}+B_{\|} \varpi \mathcal{A}_{3}
\end{aligned}
$$

Note that for singly charged ions, $\mathcal{A}_{1}, \mathcal{A}_{3} \rightarrow 0$ in the zeroth-order driftkinetic limit due to quasineutrality.

With these definitions, the field equations become

$$
\begin{aligned}
\Phi & =\varpi \mathcal{D} \mathcal{C}_{3}\left(\Phi \mathcal{A}_{1}+B_{\|} \mathcal{A}_{2}\right)-\varpi \mathcal{D} \mathcal{C}_{2}\left(\Phi \mathcal{A}_{2}+B_{\|} \mathcal{A}_{3}\right) \\
B_{\|} & =\varpi \mathcal{D} \mathcal{C}_{1}\left(\Phi \mathcal{A}_{2}+B_{\|} \mathcal{A}_{3}\right)-\varpi \mathcal{D C} \mathcal{C}_{2}\left(\Phi \mathcal{A}_{1}+B_{\|} \mathcal{A}_{2}\right)
\end{aligned}
$$

Therefore,

$$
B_{\|}=\frac{\Phi\left(\mathcal{C}_{1} \mathcal{A}_{2}-\mathcal{C}_{2} \mathcal{A}_{1}\right)}{(\varpi \mathcal{D})^{-1}-\mathcal{C}_{1} \mathcal{A}_{3}+\mathcal{C}_{2} \mathcal{A}_{2}}
$$

allowing for the elimination of $\Phi$, which in turn yields (with all real $\mathcal{C}$ and $\mathcal{A}$ )

$$
\begin{aligned}
(\varpi \mathcal{D})^{-1}= & \mathcal{C}_{3} \mathcal{A}_{1}+\frac{\mathcal{C}_{3} \mathcal{A}_{2}\left(\mathcal{C}_{1} \mathcal{A}_{2}-\mathcal{C}_{2} \mathcal{A}_{1}\right)}{(\varpi \mathcal{D})^{-1}-\mathcal{C}_{1} \mathcal{A}_{3}+\mathcal{C}_{2} \mathcal{A}_{2}} \\
& -\mathcal{C}_{2} \mathcal{A}_{2}-\frac{\mathcal{C}_{2} \mathcal{A}_{3}\left(\mathcal{C}_{1} \mathcal{A}_{2}-\mathcal{C}_{2} \mathcal{A}_{1}\right)}{(\varpi \mathcal{D})^{-1}-\mathcal{C}_{1} \mathcal{A}_{3}+\mathcal{C}_{2} \mathcal{A}_{2}}
\end{aligned}
$$

or, alternatively,

$$
\begin{gathered}
(\varpi \mathcal{D})^{2}\left(-\mathcal{C}_{3} \mathcal{C}_{1} \mathcal{A}_{1} \mathcal{A}_{3}+\mathcal{C}_{3} \mathcal{C}_{2} \mathcal{A}_{1} \mathcal{A}_{2}+\mathcal{C}_{3} \mathcal{C}_{1} \mathcal{A}_{2}^{2}-\mathcal{C}_{3} \mathcal{C}_{2} \mathcal{A}_{2} \mathcal{A}_{1}\right. \\
\left.+\mathcal{C}_{2} \mathcal{C}_{1} \mathcal{A}_{2} \mathcal{A}_{3}-\mathcal{C}_{2}^{2} \mathcal{A}_{2}^{2}-\mathcal{C}_{2} \mathcal{C}_{1} \mathcal{A}_{3} \mathcal{A}_{2}+\mathcal{C}_{2}^{2} \mathcal{A}_{3} \mathcal{A}_{1}\right) \\
+\varpi \mathcal{D}\left(\mathcal{C}_{1} \mathcal{A}_{3}-2 \mathcal{C}_{2} \mathcal{A}_{2}+\mathcal{C}_{3} \mathcal{A}_{1}\right)-1=0
\end{gathered}
$$

The condition for instability (i.e., for $\operatorname{Im}(\varpi) \neq 0$ ) therefore becomes

$$
\begin{gathered}
-4\left(-\mathcal{C}_{3} \mathcal{C}_{1} \mathcal{A}_{1} \mathcal{A}_{3}+\mathcal{C}_{3} \mathcal{C}_{2} \mathcal{A}_{1} \mathcal{A}_{2}+\mathcal{C}_{3} \mathcal{C}_{1} \mathcal{A}_{2}^{2}-\mathcal{C}_{3} \mathcal{C}_{2} \mathcal{A}_{2} \mathcal{A}_{1}\right. \\
\left.+\mathcal{C}_{2} \mathcal{C}_{1} \mathcal{A}_{2} \mathcal{A}_{3}-\mathcal{C}_{2}^{2} \mathcal{A}_{2}^{2}-\mathcal{C}_{2} \mathcal{C}_{1} \mathcal{A}_{3} \mathcal{A}_{2}+\mathcal{C}_{2}^{2} \mathcal{A}_{3} \mathcal{A}_{1}\right) \\
>\left(\mathcal{C}_{1} \mathcal{A}_{3}-2 \mathcal{C}_{2} \mathcal{A}_{2}+\mathcal{C}_{3} \mathcal{A}_{1}\right)^{2}
\end{gathered}
$$


From evaluation at typical parameters (here, $\omega_{n}=B_{0}=T_{\mathrm{e} 0} / T_{\mathrm{i} 0}=1$ and $k_{y}=\beta=0.01$, hydrogen mass ratio), one can compare the constituting quantities:

$$
\mathcal{C}_{1} \approx 1.0 \times 10^{-4} \quad \mathcal{C}_{2} \approx 1.5 \times 10^{-4} \quad \mathcal{C}_{3} \approx-204.0 \quad \mathcal{D} \approx-49.0
$$

meaning $-2 / \beta \approx \mathcal{C}_{3} \gg \mathcal{C}_{1} \sim \mathcal{C}_{2}$. Similarly,

$$
\mathcal{A}_{1} \approx-1.0 \times 10^{-4} \quad \mathcal{A}_{2} \approx 2.0 \quad \mathcal{A}_{3} \approx-3.0 \times 10^{-4}
$$

Thus, the magnitude of the relevant terms can be estimated:

$$
\begin{array}{ccc}
\mathcal{C}_{3} \mathcal{C}_{1} \mathcal{A}_{1} \mathcal{A}_{3} \sim 10^{-9} & \mathcal{C}_{3} \mathcal{C}_{2} \mathcal{A}_{1} \mathcal{A}_{2} \sim 10^{-5} \quad \mathcal{C}_{3} \mathcal{C}_{1} \mathcal{A}_{2}^{2} \sim 10^{-1} \\
\mathcal{C}_{3} \mathcal{C}_{2} \mathcal{A}_{2} \mathcal{A}_{1} \sim 10^{-5} & \mathcal{C}_{2} \mathcal{C}_{1} \mathcal{A}_{2} \mathcal{A}_{3} \sim 10^{-11} \quad \mathcal{C}_{2}^{2} \mathcal{A}_{2}^{2} \sim 10^{-7} \\
\mathcal{C}_{2} \mathcal{C}_{1} \mathcal{A}_{3} \mathcal{A}_{2} \sim 10^{-11} \quad \mathcal{C}_{2}^{2} \mathcal{A}_{3} \mathcal{A}_{1} \sim 10^{-15} \\
\mathcal{C}_{1} \mathcal{A}_{3} \sim 10^{-8} \quad \mathcal{C}_{2} \mathcal{A}_{2} \sim 10^{-4} \quad \mathcal{C}_{3} \mathcal{A}_{1} \sim 10^{-2}
\end{array}
$$

Clearly, the third term dominates the condition for instability in magnitude. As it has negative sign, the condition is fulfilled. Examining the second-to-last term in Eq. (A26) in relation to the third, one can also see that $\operatorname{Im}(\varpi) \gg \operatorname{Re}(\varpi)$. With this ordering, the equation for $\varpi$ reduces to

$$
(\varpi \mathcal{D})^{2} \mathcal{C}_{3} \mathcal{C}_{1} \mathcal{A}_{2}^{2}+\varpi \mathcal{D} \mathcal{C}_{3} \mathcal{A}_{1}-1=0
$$

The solution is therefore

$$
\varpi=\frac{-\mathcal{C}_{3} \mathcal{A}_{1} \pm 2\left(\mathcal{C}_{3} \mathcal{C}_{1} \mathcal{A}_{2}^{2}\right)^{1 / 2}}{2 \mathcal{D C}_{3} \mathcal{C}_{1} \mathcal{A}_{2}^{2}}=-2.55 \times 10^{-3} \pm 0.0714 i
$$

for the parameter choice mentioned above, which translates to

$$
\gamma, \omega=0.1399,0.004996
$$

By comparison, solving the full gyrokinetic dispersion relation in Maple yields a very similar

$$
\gamma, \omega=0.1399,0.004848
$$

which, in turn, is nearly identical with the gyrokinetic simulation results from GENE simulations,

$$
\gamma, \omega=0.1398,0.004844
$$


and runs performed with AstroGK,

$$
\gamma, \omega=0.1397,0.004851
$$

Note that the latter are shown here in GENE normalization.

To obtain parametric dependencies as applicable near the above point in parameters space, one may write the complex frequency components as

$$
\gamma \approx \frac{\omega_{n} k_{y}}{\operatorname{Im}(\varpi)} \quad \omega=-\frac{\omega_{n} k_{y} \operatorname{Re}(\varpi)}{\operatorname{Im}(\varpi)^{2}}
$$

and thus

$$
\begin{aligned}
\gamma & =\omega_{n} k_{y}|\mathcal{D}|\left(-\mathcal{C}_{3} \mathcal{C}_{1} \mathcal{A}_{2}^{2}\right)^{1 / 2} \\
\omega & =-\frac{1}{2} \omega_{n} k_{y} \mathcal{D} \mathcal{C}_{3} \mathcal{A}_{1}
\end{aligned}
$$

based on Eq. (A31). Writing out the constituting terms gives the following expressions:

$$
\begin{aligned}
\gamma= & \omega_{n} k_{y}\left(\frac{2}{\beta}+\sum_{j} \frac{2 n_{j 0} T_{j 0}}{B_{0}^{2}}\left(\Gamma_{0}-\Gamma_{1}\right)\right)^{1 / 2}\left(\sum_{j} \frac{n_{j 0} T_{j 0}}{B_{0}}\left(\Gamma_{0}-\Gamma_{1}\right)\right) \\
& \times\left(\sum_{j} \frac{q_{j}^{2} n_{j 0}}{T_{j 0}}\left(1-\Gamma_{0}\right)\right)^{1 / 2} /\left[\sum_{j} \frac{q_{j}^{2} n_{j 0}}{T_{j 0}}\left(1-\Gamma_{0}\right)\right) \\
& \left.\times\left(\frac{2}{\beta}+\sum_{j} \frac{2 n_{j 0} T_{j 0}}{B_{0}^{2}}\left(\Gamma_{0}-\Gamma_{1}\right)\right)+\left(\sum_{j} \frac{q_{j} n_{j 0}}{B_{0}}\left(\Gamma_{0}-\Gamma_{1}\right)\right)^{2}\right] \\
\omega= & \frac{\omega_{n} k_{y}}{2}\left(-\frac{2}{\beta}-\sum_{j} \frac{2 n_{j 0} T_{j 0}}{B_{0}^{2}}\left(\Gamma_{0}-\Gamma_{1}\right)\right) \\
& \times\left(\sum_{j} q_{j} n_{j 0} \Gamma_{0}\right) /\left(\sum_{j} \frac{q_{j}^{2} n_{j 0}}{T_{j 0}}\left(1-\Gamma_{0}\right)\right)^{2} \\
& \left.\times\left(-\frac{2}{\beta}-\sum_{j} \frac{2 n_{j 0} T_{j 0}}{B_{0}^{2}}\left(\Gamma_{0}-\Gamma_{1}\right)\right)-\left(\sum_{j} \frac{q_{j} n_{j 0}}{B_{0}}\left(\Gamma_{0}-\Gamma_{1}\right)\right)^{2}\right]
\end{aligned}
$$

which, by comparing the different $\mathcal{C}$ and using $\mathcal{C}_{3}=-2 / \beta$ (in the low- $\beta$ limit), can be simplified to

$$
\begin{aligned}
& \gamma=\omega_{n} k_{y}\left(\frac{2}{\beta}\right)^{-1 / 2}\left(\sum_{j} \frac{n_{j 0} T_{j 0}}{B_{0}}\left(\Gamma_{0}-\Gamma_{1}\right)\right)\left(\sum_{j} \frac{q_{j}^{2} n_{j 0}}{T_{j 0}}\left(1-\Gamma_{0}\right)\right)^{-1 / 2} \\
& \omega=-\frac{\omega_{n} k_{y}}{2}\left(\sum_{j} q_{j} n_{j 0} \Gamma_{0}\right)\left(\sum_{j} \frac{q_{j}^{2} n_{j 0}}{T_{j 0}}\left(1-\Gamma_{0}\right)\right)^{-1}
\end{aligned}
$$


In the low- $k_{y}$, driftkinetic limit, one can write (with $b_{j}=k_{y}^{2} T_{j 0} m_{j} /\left(q_{j}^{2} B_{0}^{2}\right)$ )

$$
\Gamma_{0} \approx 1-b_{j} \quad 1-\Gamma_{0} \approx b_{j} \quad \Gamma_{0}-\Gamma_{1} \approx 1-\frac{3}{2} b_{j} \approx 1
$$

where the linear contribution has to be retained in the first expression because of the zeroorder term canceling due to quasineutrality, see below; whereas in the last expression the linear contribution - which is quadratic in $k_{y}$ - is dominated by the zero-order term. Therefore

$$
\begin{aligned}
& \gamma=\omega_{n} k_{y}\left(\frac{2}{\beta}\right)^{-1 / 2}\left(\sum_{j} \frac{n_{j 0} T_{j 0}}{B_{0}}\right)\left(\sum_{j} k_{y}^{2} \frac{n_{j 0} m_{j}}{B_{0}^{2}}\right)^{-1 / 2} \\
& \omega=-\frac{\omega_{n} k_{y}}{2}\left(\sum_{j} q_{j} n_{j 0}\left(1-k_{y}^{2} \frac{T_{j 0} m_{j}}{q_{j}^{2} B_{0}^{2}}\right)\right)\left(\sum_{j} k_{y}^{2} \frac{n_{j 0} m_{j}}{B_{0}^{2}}\right)^{-1}
\end{aligned}
$$

Since $\sum_{j} q_{j} n_{j 0}=0$ due to quasineutrality, one can write

$$
\begin{aligned}
& \gamma=\omega_{n}\left(\frac{2}{\beta}\right)^{-1 / 2}\left(\sum_{j} n_{j 0} T_{j 0}\right)\left(\sum_{j} n_{j 0} m_{j}\right)^{-1 / 2} \\
& \omega=\frac{\omega_{n} k_{y}}{2}\left(\sum_{j} \frac{n_{j 0} T_{j 0} m_{j}}{q_{j}}\right)\left(\sum_{j} n_{j 0} m_{j}\right)^{-1}
\end{aligned}
$$

These expressions are valid to first order in $k_{y}$. With a hydrogen plasma $\left(q_{\mathrm{i}}=-q_{\mathrm{e}}\right.$ and $\left.m_{\mathrm{e}} \ll m_{\mathrm{i}}\right)$ and assuming $T_{\mathrm{i} 0} / T_{\mathrm{e} 0} \gg m_{\mathrm{e}} / m_{\mathrm{i}}$, this becomes

$$
\begin{aligned}
& \gamma=\omega_{n}\left(\frac{\beta n_{\mathrm{i} 0}}{2 m_{\mathrm{i}}}\right)^{1 / 2}\left(T_{\mathrm{e} 0}+T_{\mathrm{i} 0}\right) \quad \underset{m, n, T}{\mathrm{insert}} \quad \omega_{n} \sqrt{2 \beta}=0.14 \\
& \omega=\frac{\omega_{n} k_{y}}{2} \frac{T_{\mathrm{i} 0}}{q_{\mathrm{i}}} \quad \frac{\text { insert }}{m, n, T} \quad \frac{\omega_{n} k_{y}}{2}=0.005
\end{aligned}
$$

in good agreement with the aforementioned results.

In summary,

$$
\gamma \propto \omega_{n} k_{y}^{0} \beta^{1 / 2} \quad \omega \propto \omega_{n} k_{y} \beta^{0}
$$

Note that both analytical and simulation approaches using zeroth-order (in $k_{y}$ ) approximations for $J_{0}$ and $J_{1}$ yield different results for the frequencies.

[1] B.N. Rogers, S. Kobayashi, P. Ricci, W. Dorland, J. Drake, and T. Tatsuno, Phys. Plasmas 14, 092110 (2007) 
[2] J.F. Drake, M. Swisdak, T.D. Phan, P.A. Cassak, M.A. Shay, S.T. Lepri, R.P. Lin, E. Quataert, and T.H. Zurbuchen, J. Geophys. Res. 114, 05111 (2009)

[3] W. Daughton, V. Roytershteyn, B.J. Albright, H. Karimabadi, L. Yin, and K.J. Bowers, Phys. Rev. Lett. 103, 065004 (2009)

[4] E.G. Zweibel, E. Lawrence, J. Yoo, H. Ji, M. Yamada, and L.M. Malyshkin, Phys. Plasmas 18, $111211(2011)$

[5] M.J. Pueschel, F. Jenko, D. Told, and J. Büchner, Phys. Plasmas 18, 112102 (2011)

[6] R. Numata, W. Dorland, G.G. Howes, N.F. Loureiro, B.N. Rogers, and T. Tatsuno, Phys. Plas$\operatorname{mas} \mathbf{1 8}, 112106(2011)$

[7] J.W. Connor, S.C. Cowley, R.J. Hastie, T.C. Hender, A. Hood, and T.J. Martin, Phys. Fluids 31, 577 (1988)

[8] H. Doerk, F. Jenko, M.J. Pueschel, and D.R. Hatch, Phys. Rev. Lett. 106, 155003 (2011)

[9] W. Guttenfelder, J. Candy, S.M. Kaye, W.M. Nevins, E. Wang, R.E. Bell, G.W. Hammett, B.P. LeBlanc, D.R. Mikkelsen, and H. Yuh, Phys. Rev. Lett. 106, 155004 (2011)

[10] H. Doerk, F. Jenko, T. Görler, D. Told, M.J. Pueschel, and D.R. Hatch, Phys. Plasmas 19, 055907 (2012)

[11] M. Swisdak, B.N. Rogers, J.F. Drake, and M.A. Shay, J. Geophys. Res. 103, 1218 (2003)

[12] E. Tassi, F.L. Waelbroeck, and D. Grasso, J. Phys. Conf. Series 260, 012020 (2010)

[13] D. Grasso, F.L. Waelbroeck, and E. Tassi, J. Phys. Conf. Series 401, 012008 (2012)

[14] O. Zacharias, R. Kleiber, and R. Hatzky, J. Phys. Conf. Series 401, 012026 (2012)

[15] J.W. Connor, R.J. Hastie, and A. Zocco, Plasma Phys. Control. Fusion 54, 035003 (2012)

[16] F. Porcelli, Phys. Rev. Lett. 66, 425 (1991)

[17] F. Jenko, W. Dorland, M. Kotschenreuther, and B.N. Rogers, Phys. Plasmas 7, 1904 (2000)

[18] see http://genecode.org for code details and access

[19] A.J. Brizard and T.S. Hahm, Rev. Mod. Phys. 79, 421 (2007)

[20] R. Numata, G.G. Howes, T. Tatsuno, M. Barnes, and W. Dorland, J. Comp. Phys. 229, 9347 (2010)

[21] E.A. Belli and J. Candy, Phys. Plasmas 17, 112314 (2010)

[22] T. H. Dupree, Phys. Fluids 9, 1773 (1966)

[23] W. Horton and D. Choi, Phys. Reports 49, 273 (1979)

[24] M.J. Pueschel, D. Told, P.W. Terry, F. Jenko, E.G. Zweibel, V. Zhdankin, and H. Lesch, 
Astrophys. J. Suppl. Ser. 213, 30 (2014)

[25] P.A. Cassak, J.F. Drake, and M.A. Shay, Astrophys. J. 644, L145 (2006)

[26] E.R. Priest and T.G. Forbes, Astron. Astrophys. Rev. 10, 313 (2002)

[27] A.F. Rappazzo and M. Velli, Phys. Rev. E 83, 065401 (2011)

[28] E.N. Parker, Astrophys. J. 330, 474 (1988) 